\title{
Breatherlike excitations in discrete lattices with noise and nonlinear damping
}

\author{
Christiansen, Peter Leth; Gaididei, Yuri B.; Johansson, Magnus; Rasmussen, Kim
}

Published in:

Physical Review B

Link to article, DOI:

10.1103/PhysRevB.55.5759

Publication date:

1997

Document Version

Publisher's PDF, also known as Version of record

Link back to DTU Orbit

Citation (APA):

Christiansen, P. L., Gaididei, Y. B., Johansson, M., \& Rasmussen, K. (1997). Breatherlike excitations in discrete lattices with noise and nonlinear damping. Physical Review B, 55(9), 5759-5766.

https://doi.org/10.1103/PhysRevB.55.5759

\section{General rights}

Copyright and moral rights for the publications made accessible in the public portal are retained by the authors and/or other copyright owners and it is a condition of accessing publications that users recognise and abide by the legal requirements associated with these rights.

- Users may download and print one copy of any publication from the public portal for the purpose of private study or research.

- You may not further distribute the material or use it for any profit-making activity or commercial gain

- You may freely distribute the URL identifying the publication in the public portal 


\title{
Breatherlike excitations in discrete lattices with noise and nonlinear damping
}

\author{
Peter L. Christiansen \\ Department of Mathematical Modelling, The Technical University of Denmark, DK-2800 Lyngby, Denmark
}

Yuri B. Gaididei

Institute for Theoretical Physics, Metrologicheskaya Street 14 B, 252143 Kiev 143, Ukraine

Magnus Johansson and Kim $\emptyset$. Rasmussen

Department of Mathematical Modelling, The Technical University of Denmark, DK-2800 Lyngby, Denmark

(Received 30 July 1996)

\begin{abstract}
We discuss the stability of highly localized, "breatherlike," excitations in discrete nonlinear lattices under the influence of thermal fluctuations. The particular model considered is the discrete nonlinear Schrödinger equation in the regime of high nonlinearity, where temperature effects are included as multiplicative white noise and nonlinear damping. Numerical analysis shows that the lifetime of the breather is always finite and, in a large parameter regime, inversely proportional to the noise variance for fixed damping and nonlinearity. We also find that the decay rate of the breather decreases with increasing nonlinearity and with increasing damping. Using a collective-coordinate approximation, we show how the qualitative features of the numerical results can be analytically understood. Finally, in the dimer case we show that the multiplicative noise can be transformed into additive noise, and an exact stationary solution to the Fokker-Planck equation is obtained. From this solution, the dimer system is found to exhibit a noise (temperature) induced phase transition. [S0163-1829(97)07409-2]
\end{abstract}

\section{INTRODUCTION}

The existence of highly localized, time-periodic solutions in lattice equations with large nonlinearity is by now a well established fact. ${ }^{1-4}$ These solutions have been termed intrinsically localized modes, reflecting the fact that no external defects are needed for their creation, or discrete breathers, in analogy with the well-known solutions to integrable nonlinear partial differential equations, which have similar properties. In the particular case of the one-dimensional (1D) discrete nonlinear Schrödinger (DNLS) equation, which will be considered in this paper, the creation of these modes is an example of the well-known discrete self-trapping (DST) phenomenon, ${ }^{5-7}$ by which an initially localized excitation remains localized around the initially excited site for all times. For this reason, a class of equations including the DNLS equation has also been termed the DST equation. ${ }^{5}$ The DST phenomenon has been used to model energy localization in a large number of physical contexts, such as polaron formation in electron-lattice coupled systems, ${ }^{8}$ localization of vibrational energy in proteins, ${ }^{9}$ and localization of optical beams in nonlinear waveguide arrays. ${ }^{10}$ The discrete breathers have also been found ${ }^{11-13}$ to play an important part as final states in the "quasicollapse" process, which is the discrete analog to the ordinary collapse phenomenon occurring in the continuum nonlinear Schrödinger (NLS) equation in dimensions larger than 1 or with higher degree nonlinearities. ${ }^{14}$ Since the 2D DNLS equation also has been used to describe exciton dynamics in models for so-called Scheibe aggregates in molecular thin films, ${ }^{15,16}$ the discrete breathers may be of importance also in this context.

A frequently used method to take into account effects of finite temperature in models describing a quantum quasiparticle (electron or exciton) interacting with lattice vibrations is to add white noise and damping to the lattice equations, ${ }^{16-18}$ thereby turning these into Langevin equations. As was shown in Ref. 16, the coupled exciton-phonon equations can under certain approximations be reduced to a single DNLS equation describing the exciton dynamics, where the effects of thermal fluctuations of the phonons appear as a multiplicative noise term. The spectrum of the noise will then in general be colored, but by assuming short correlation times, it can be treated as white in a first approximation. However, as was recently shown, ${ }^{19}$ in order to take proper account of the damping of the phonon system, it is necessary (at least when the white-noise approximation is made) to also include a nonlinear damping term in the exciton equation. With this inclusion, it was shown that under certain conditions an energy balance may be established in the exciton system.

In this paper, we will consider the 1D DNLS equation with multiplicative noise and nonlinear damping included, and investigate how the inclusion of these terms affects the discrete breathers. The restriction to $1 \mathrm{D}$ is mainly for numerical convenience; similar results are expected also in two and three dimensions. Results for the numerical integration of the resulting system of stochastic differential equations are discussed in Sec. II, while in Sec. III a collectivecoordinate approach is used to obtain approximate analytical results. In Sec. IV we discuss the particular case when the system only contains two sites (the DNLS dimer), for which some exact analytical results can be obtained. Finally, Sec. V contains some concluding remarks.

\section{NUMERICAL ANALYSIS}

The form of the DNLS equation to be considered here is

$$
i \dot{\psi}_{n}+J\left(\psi_{n+1}+\psi_{n-1}\right)+\gamma\left|\psi_{n}\right|^{2} \psi_{n}-\eta \psi_{n} \frac{d}{d t}\left[\left|\psi_{n}(t)\right|^{2}\right]
$$




$$
+h_{n}(t) \psi_{n}=0
$$

where the last two terms describe nonlinear damping and multiplicative Gaussian white noise, respectively. The noise is assumed to have zero mean and variance $2 D$, i.e.,

$$
\left\langle h_{n}(t)\right\rangle=0, \quad\left\langle h_{n}(t) h_{n^{\prime}}\left(t^{\prime}\right)\right\rangle=2 D \delta\left(t-t^{\prime}\right) \delta_{n n^{\prime}} .
$$

As was shown in Ref. 19, Eq. (1) can be derived from the following equations modeling a quasiparticle (e.g., electron or exciton) interacting with an optical phonon field in contact with a heat bath (in units with $\hbar=1$ ):

$$
\begin{gathered}
i \dot{\psi}_{n}+J\left(\psi_{n+1}+\psi_{n-1}\right)+\chi u_{n} \psi_{n}=0, \\
M \ddot{u}_{n}+M \lambda \dot{u}_{n}+M \omega_{0}^{2} u_{n}-\chi\left|\psi_{n}\right|^{2}=\eta_{n}(t) .
\end{gathered}
$$

Here $\psi_{n}$ is the complex amplitude of the quasiparticle wave function at site $n$ and $u_{n}$ represents the elastic degree of freedom at site $n$. Furthermore, $J$ is the nearest-neighbor hopping constant, $\chi$ is the coupling constant between the quasiparticle and the phonons, $M$ is the molecular mass, $\lambda$ is a damping coefficient, $\omega_{0}$ is the Einstein frequency of each oscillator, and $\eta_{n}(t)$ is a stochastic force acting on the phonon system. The variance of the stochastic force should be related to the temperature $T$ and the damping coefficient $\lambda$ according to the fluctuation-dissipation theorem in order to drive the system into thermal equilibrium. Under the assumptions made in Refs. 16 and 19, the most important being that the quasiparticle field should vary slowly in time compared with the lattice vibrations, Eq. (1) was obtained ${ }^{19}$ with the following relations between the parameters:

$$
\gamma=\frac{\chi^{2}}{M \omega_{0}^{2}}, \quad \eta=\gamma \frac{\lambda}{\omega_{0}^{2}}, \quad D=\eta k_{B} T,
$$

where $k_{B}$ is Boltzmann's constant.

It is important to note that also in the presence of noise and nonlinear damping, the DNLS equation (1) still has one conserved quantity, namely, the norm (excitation number) $\mathcal{N}$ of the solution defined as

$$
\mathcal{N}=\sum_{n}\left|\psi_{n}\right|^{2} .
$$

However, the Hamiltonian $\mathcal{H}$, defined as

$$
\mathcal{H}=-J \sum_{n}\left(\psi_{n} \psi_{n+1}^{*}+\psi_{n}^{*} \psi_{n+1}\right)-\frac{\gamma}{2} \sum_{n}\left|\psi_{n}\right|^{4},
$$

which is the second conserved quantity for the unperturbed DNLS equation, will in the presence of noise and damping, as in Eq. (1), no longer be conserved. Instead, its time evolution will be determined by

$$
\frac{d \mathcal{H}}{d t}=-\eta \sum_{n}\left(\frac{d}{d t}\left(\left|\psi_{n}\right|^{2}\right)\right)^{2}+\sum_{n} h_{n}(t) \frac{d}{d t}\left(\left|\psi_{n}\right|^{2}\right)
$$

Considering the ensemble average of this equation, we find that the damping and noise terms will in average provide dissipation and input of energy to the system, respectively.

In our numerical simulations, we have without loss of generality chosen the quasiparticle wave function to be normalized, i.e., $\mathcal{N}=1$ (results for arbitrary $\mathcal{N}$ are simply ob-

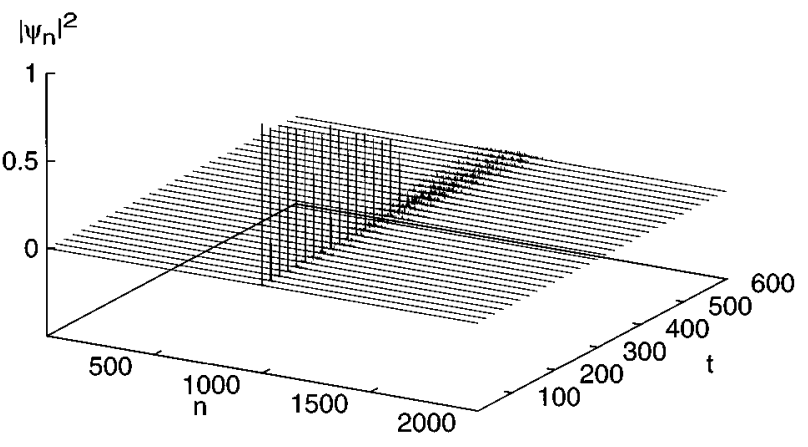

FIG. 1. Time evolution of an initially single-site localized excitation. Parameter values are $\gamma=10, \eta=2$, and $D=0.05$.

tained by the rescaling $\gamma \rightarrow \gamma \mathcal{N}, \eta \rightarrow \eta \mathcal{N})$. Furthermore, we define the energy (or time) scale by fixing the hopping constant to $J=1$. Finally, we choose the initial condition to be a single-site excitation at site $n_{0}$, i.e.,

$$
\psi_{n}(0)=\delta_{n, n_{0}}
$$

With this choice of initial condition, it has previously been found ${ }^{6,7,20}$ that in the absence of noise and damping the DNLS equation exhibits a self-trapping transition when $\gamma=\gamma_{c} \approx 3.5$, so that when $\gamma>\gamma_{c}$ the initial-site probability $\left|\psi_{n_{0}}\right|^{2}$ will always be nonzero. As $\gamma$ is increased beyond $\gamma_{c}$, the total norm of the excitation trapped around the initial site increases and the width of the excitation decreases. In the calculations reported here, we consider nonlinearities $\gamma \geqslant 5$, for which the trapped excitation has a highly discrete breatherlike nature (for $\gamma=5$, the breather is mainly localized on the initial site and its two neighboring sites, and the stationary value of the initial-site probability in the absence of noise and damping is found to be approximately 0.77 ).

An illustration of how the presence of noise in Eq. (1) affects the DNLS breather is given in Fig. 1. As the figure shows, the main effect of the noise is initially to cause a slow decrease of the breather amplitude. This slow decrease continues until the initial-site probability has reached approximately half of its initial value, at which point the breather is rapidly destroyed. The long-time behavior is then found to be diffusive, similar to what is seen in the linear system $(\gamma=0)$. The result presented in Fig. 1, as well as all other numerical results reported here, has been obtained by numerical integration of Eq. (1) using a stochastic version of the fourth-order Runge-Kutta-Merson algorithm, ${ }^{21,22}$ making sure that the statistical properties of the solution remain invariant under changes of the size of the time steps.

By a systematic investigation of the numerical solutions to Eq. (1) for different values of $\gamma, \eta$, and $D$, we have found that the behavior shown in Fig. 1 appears to be generic. The lifetime of the breather is always finite, but increases when (a) the nonlinearity $\gamma$ is increased, (b) the nonlinear damping $\eta$ is increased, or (c) the noise variance $D$ is decreased. This can be seen from Fig. 2, which shows some typical examples of how the initial-site probability varies with time for different values of the parameters. (In all cases, we have made sure that the system size is large enough, so that boundary effects can be neglected in the time regime shown in the figures.) The typical scenario is the following: After a short 

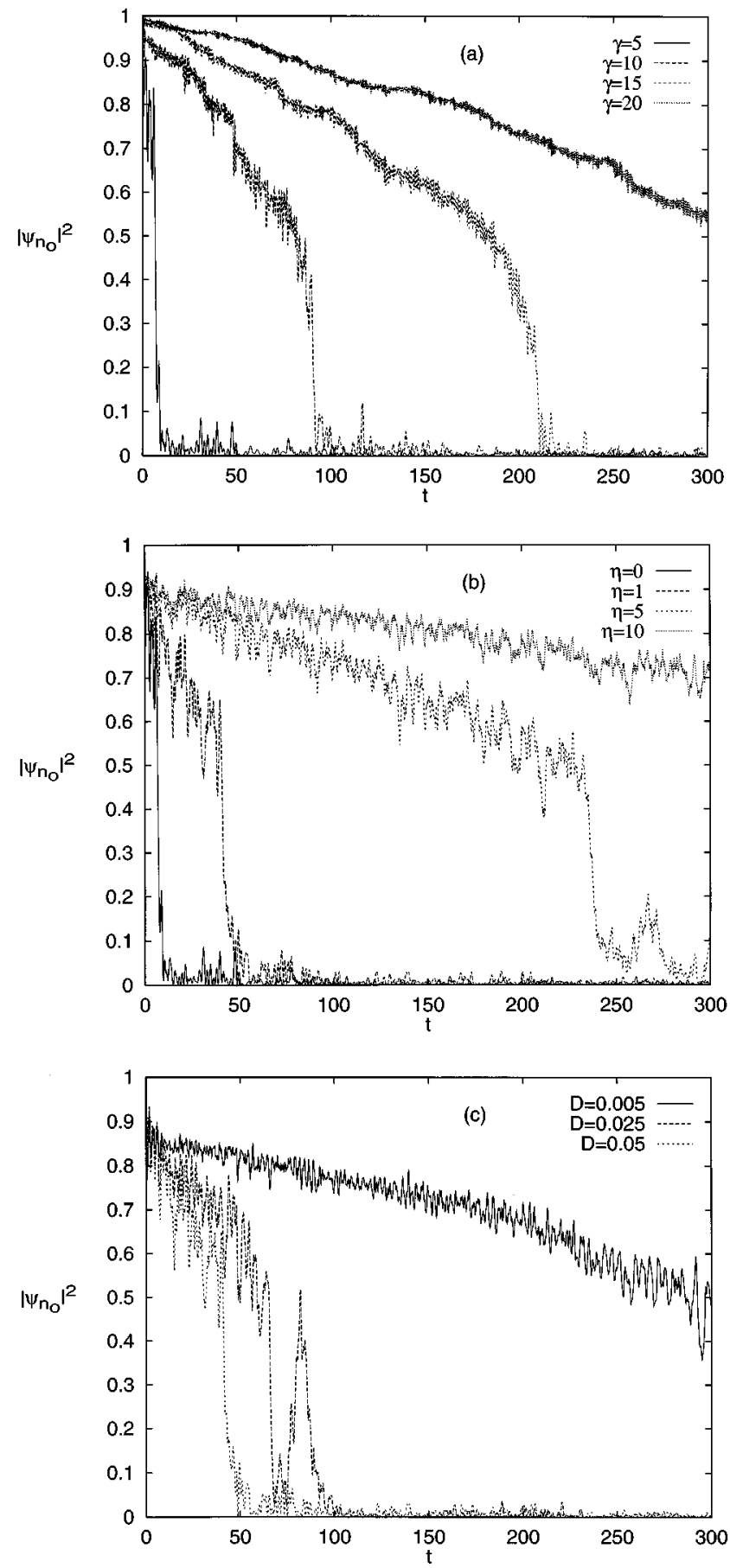

FIG. 2. Typical time evolution of the initial-site probability for some different values of $\gamma, \eta$, and $D$. In (a) we have $\eta=0, D=0.05$, and from bottom to top $\gamma=5,10,15$, and 20; in (b), $\gamma=5, D=0.05$, and from bottom to top $\eta=0,1,5$, and 10 ; in (c), $\gamma=5, \eta=1.0$, and $D=0.005,0.025$, and 0.05 from top to bottom. The particular realization of the noise is the same in all cases.

initial time interval, where the breather is created and $\left|\psi_{n_{0}}\right|^{2}$ rapidly drops to a value close to its stationary value in the absence of noise, the initial-site probability decays almost linearly with time (this is seen even more clearly if averaging over different noise realizations is performed). This linear decay continues until the value of $\left|\psi_{n_{0}}\right|^{2}$ has been reduced by approximately a factor $1 / 2$, when the initial-site probability rapidly drops to values close to zero, and the breather is destroyed. From this point on, the system behaves approximately as a linear system (i.e., diffusively), and the initial-site probability decays in average as $t^{-1 / 2}$ just as for an ordinary random-walk process.

The results from a more quantitative numerical analysis of the decay rate of the breather as a function of the parameters $\gamma, \eta$, and $D$, obtained by averaging over different realizations of the noise, are displayed in Fig. 3. The decay rate $\kappa$ is here defined as the mean value of $(-d / d t)\left(\left|\psi_{n_{0}}\right|^{2}\right)$ in the time interval of almost linear decay. [From the values of $\kappa$, the approximate lifetime $t_{L}$ of the breather can then be obtained as $t_{L} \approx 1 /(2 \kappa)$, as discussed above.] From the results shown in Fig. 3(a), as well as from similar results obtained for a number of other values of the parameters, we conclude that the decay rate appears to be proportional to the variance of the noise over a large parameter region. (Although no error bars are plotted in Fig. 3, we estimate the maximum error in each data point to be approximately $5-10 \%$. The errors are mainly due to the computational limits of the number of samples used in the averaging procedures.) We found this proportionality to be valid as long as the noise is weak enough not to affect the creation of the breather considerably (the upper limit on $D$ for this regime depends on $\gamma$ and $\eta$ ). If the noise is too strong, no breatherlike state will be created, and the diffusive spreading starts immediately.

Also, as is shown in Fig. 3(b), the decay rate is for fixed $D$ and $\eta$ approximately proportional to $\gamma^{-2}$ in the studied parameter range, while the data in Fig. 3(c) showing the variation of $\kappa$ with $\eta$ do not seem to follow any simple scaling law. As we will see in the next section, the numerically found dependence of the decay rate on $D$ and $\gamma$ agrees qualitatively with the approximate analytical results that can be obtained using the method of collective coordinates.

\section{COLLECTIVE COORDINATE APPROACH}

In this section, we will use the method of collective coordinates in order to gain some analytical understanding for how the presence of noise and nonlinear damping will affect the breather solutions to Eq. (1). The starting point of this method is to choose a localized self-similar trial function which is close to the exact solution in the absence of noise and damping, and contains a number of parameters which become time dependent due to the perturbations. In our case, we find a trial function of the form

$$
\psi_{n}(t)=A(t) e^{i \alpha(t) n^{2}-\beta(t)|n|}
$$

to be appropriate. Here the parameter $A$ determines the complex amplitude of the wave function, while the real parameters $\alpha$ and $\beta$ determine the phase chirp and width of the wave function, respectively. (The initial site is here chosen for convenience as $n_{0}=0$, without loss of generality.) This choice of trial function is motivated by the fact that in the absence of noise, the exact stationary breather solution to Eq. (1) becomes exponentially decaying in the high-nonlinearity regime. ${ }^{2}$ Thus, with $\alpha=0$, our trial function approaches in the limit of large nonlinearity the exact breather solution to the noise-free DNLS equation. The particular form of phase 

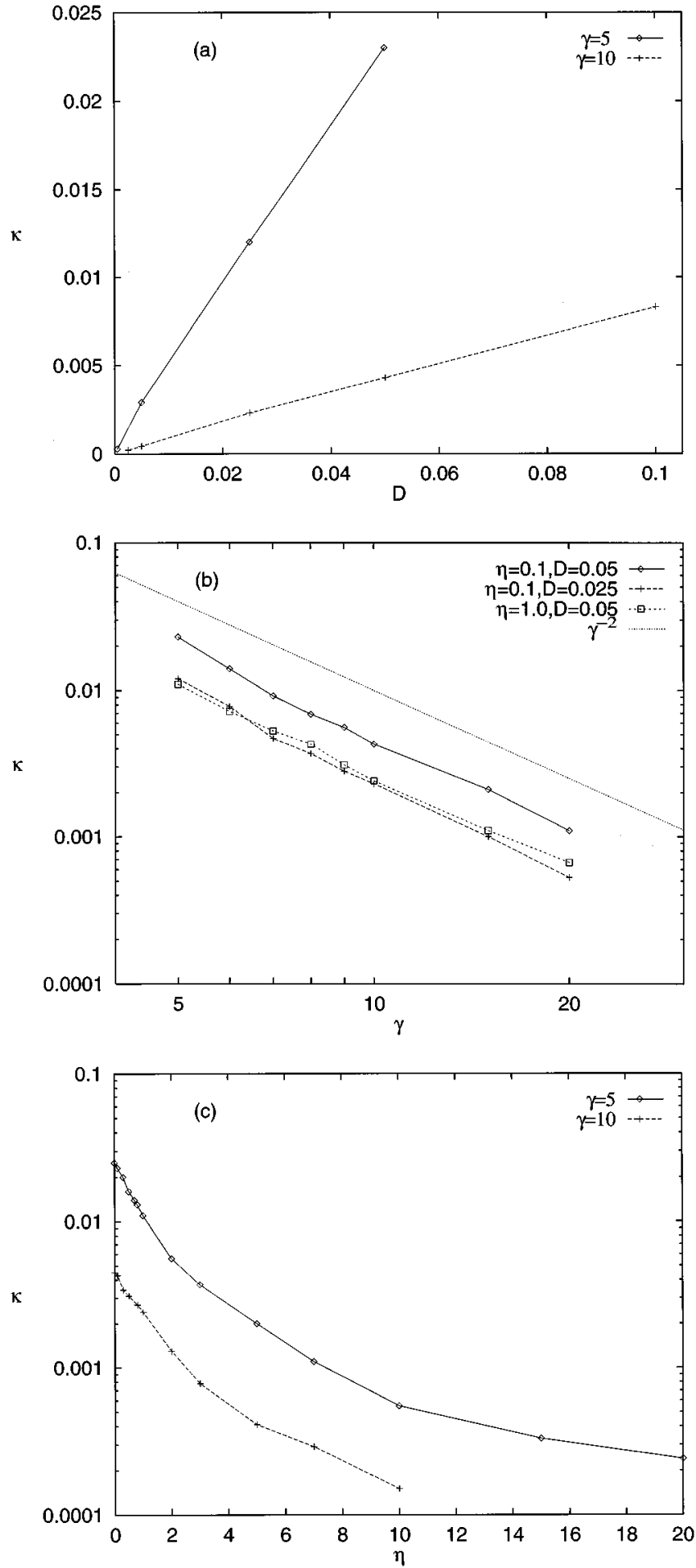

FIG. 3. Quantitative dependence of the decay rate of the breather $\kappa$ as a function of (a) noise strength $D$, (b) nonlinearity parameter $\gamma$, and (c) nonlinear damping parameter $\eta$. In (a), $\eta=0.1$ for both curves, and in (c), $D=0.05$ for both curves; other parameter values are as indicated in the figures.

chirp included in Eq. (10) was mainly inspired by previous work on the continuous NLS equation, where collective coordinate approaches using this kind of phase chirp have turned out to be successful. ${ }^{24,25,19}$ In the presence of weak noise, we can expect Eq. (10) to give an approximate representation of the breather in the time regime following shortly after its creation, i.e., when the decoherence caused by the noise is still small enough to be neglected. Using the normalization condition $\mathcal{N}=1$, with $\mathcal{N}$ defined as in Eq. (6), the relation between the parameters $A$ and $\beta$ is found to be

$$
|A(t)|^{2}=\tanh \beta(t) .
$$

In order to derive differential equations determining the time evolution of the remaining two independent parameters, we choose to work with the variable $q$ instead of $\beta$, where $q$ is defined as

$$
q(t)=\frac{1}{2 \sinh ^{2} \beta(t)} .
$$

We will first consider the case when the nonlinear damping $\eta$ is zero. In this case, we can use the Lagrangian $L$,

$$
\begin{aligned}
L= & \frac{i}{2} \sum_{n}\left(\dot{\psi}_{n} \psi_{n}^{*}-\dot{\psi}_{n}^{*} \psi_{n}\right)+J \sum_{n}\left(\psi_{n} \psi_{n+1}^{*}+\psi_{n}^{*} \psi_{n+1}\right) \\
& +\frac{\gamma}{2} \sum_{n}\left|\psi_{n}\right|^{4}+\sum_{n} h_{n}(t)\left|\psi_{n}\right|^{2}
\end{aligned}
$$

to derive a pair of coupled first-order differential equations for $\alpha$ and $q$ using the Euler-Lagrange equations. After a subsequent transformation of the noise term, which leaves the Fokker-Planck equation for the relevant probability density invariant and therefore gives an equivalent description of the process (similar transformations were performed in Refs. 19 and 25), the two equations take the form

$$
\begin{gathered}
\dot{q}=\frac{2 J \sqrt{2 q} \sin \alpha}{\sqrt{1+2 q}} \frac{1+4 q-2 q \sin ^{2} \alpha}{\left(1+2 q \sin ^{2} a\right)^{2}} \\
\dot{\alpha}=\frac{2 J \cos (\alpha)\left(1-2 q \sin ^{2} \alpha-8 q^{2} \sin ^{2} \alpha\right)}{\sqrt{2 q}(1+2 q)^{3 / 2}\left(1+2 q \sin ^{2} \alpha\right)^{2}}-\frac{\gamma}{2} \frac{(2+q)}{(1+2 q)^{5 / 2}} \\
+\sqrt{\frac{3+q}{2(1+2 q)^{7 / 2}}} h(t)
\end{gathered}
$$

where the noise $h(t)$ now only depends on time and has the autocorrelation

$$
\left\langle h(t) h\left(t^{\prime}\right)\right\rangle=2 D \delta\left(t-t^{\prime}\right) .
$$

We note that the variables $\alpha$ and $q$ are canonically conjugated, in the sense that with the Hamiltonian $\mathcal{H}$ defined as in Eq. (7), Eqs. (14) and (15) can be written as

$$
\dot{q}=\frac{\partial \mathcal{H}}{\partial \alpha}, \quad \dot{\alpha}=-\frac{\partial \mathcal{H}}{\partial q}+\sqrt{\frac{3+q}{2(1+2 q)^{7 / 2}}} h(t),
$$

where

$$
\mathcal{H}=-\frac{2 J \sqrt{2 q} \cos \alpha}{\left(1+2 q \sin ^{2} \alpha\right) \sqrt{1+2 q}}-\frac{\gamma}{2} \frac{1+q}{(1+2 q)^{3 / 2}} .
$$

We can now also include the nonlinear damping in the collective-coordinate equations (17), by using the fact that the Hamiltonian should fulfill the conservation law (8). By explicit calculation using the trial function (10), we find that 
the energy conservation law will be fulfilled if a friction term is added to Eq. (15), so that the two equations for $q$ and $\alpha$ will take the form

$$
\begin{aligned}
\dot{q}=\frac{\partial \mathcal{H}}{\partial \alpha}, \quad \dot{\alpha}= & -\frac{\partial \mathcal{H}}{\partial q}-\frac{\eta}{2} \dot{q} \frac{3+q}{(1+2 q)^{7 / 2}} \\
& +\sqrt{\frac{3+q}{2(1+2 q)^{7 / 2}}} h(t) .
\end{aligned}
$$

Equations (19) are the basic evolution equations for the collective-coordinate parameters, and they are exact as long as the description in terms of the self-similar trial function (10) is valid. In order to obtain an analytical estimate for the decay rate of the breather, we will now consider the case of strongly localized excitations, i.e., large nonlinearity $\gamma$. In this case $\beta>1$ and, using Eq. (12), $q \ll 1$. We can thus write, instead of Eqs. (19),

$$
\begin{gathered}
\dot{q}=2 J \sqrt{2 q} \sin \alpha, \\
\dot{\alpha}=\sqrt{\frac{2}{q}} J \cos \alpha-\gamma-\frac{3}{2} \eta \dot{q}+\sqrt{\frac{3}{2}} h(t) .
\end{gathered}
$$

These equations are more easily analyzed if the variables $\alpha$ and $q$ are transformed into action-angle variables. To do this, we consider the Hamilton-Jacobi equation for the case with no damping and no noise, when Eq. (20) reduces to

$$
\dot{q}=2 J \sqrt{2 q} \sin \alpha \equiv \frac{\partial \mathcal{H}_{0}}{\partial \alpha}, \quad \dot{\alpha}=\sqrt{\frac{2}{q}} J \cos \alpha-\gamma \equiv-\frac{\partial \mathcal{H}_{0}}{\partial q},
$$

where

$$
\mathcal{H}_{0}=-2 J \sqrt{2 q} \cos \alpha+\gamma q
$$

Introducing $\alpha=\partial S / \partial q$, we obtain the Hamilton-Jacobi equation

$$
-2 J \sqrt{2 q} \cos \left(\frac{\partial S}{\partial q}\right)+\gamma q=-\frac{2 J^{2}}{\gamma}(1-\mathcal{J})
$$

where $\mathcal{J}$ is the action variable $(\mathcal{J} \geqslant 0)$. From Eq. (23) we obtain that

$$
S=\int^{q} d x \arccos \left(\frac{\gamma x+\left(2 J^{2} / \gamma\right)(1-\mathcal{J})}{2 J \sqrt{2 x}}\right),
$$

and the angle variable $w$ is determined by

$$
w=\frac{\gamma^{2}}{2 J^{2}} \frac{\partial S}{\partial \mathcal{J}}
$$

From Eqs. (23)-(25) we then get

$$
\begin{gathered}
q=\frac{2 J^{2}}{\gamma^{2}}(1+\mathcal{J}+2 \sqrt{\mathcal{J}} \sin w), \\
\cos \alpha=\frac{1+\sqrt{\mathcal{J}} \sin w}{(1+\mathcal{J}+2 \sqrt{\mathcal{J}} \sin w)^{1 / 2}},
\end{gathered}
$$

so that using Eqs. (26) and (27) we obtain, instead of Eq. (20),

$$
\begin{gathered}
\dot{\mathcal{J}}=-\frac{12 \eta J^{2}}{\gamma} \mathcal{J} \cos ^{2} w+\sqrt{6 \mathcal{J}} \cos (w) h(t) \\
\dot{w}=\gamma+\frac{6 \eta J^{2}}{\gamma}(\sqrt{\mathcal{J}}+\sin w) \cos w-\sqrt{\frac{3}{2}}\left(1+\frac{\sin w}{\sqrt{\mathcal{J}}}\right) h(t) .
\end{gathered}
$$

We see that when the nonlinearity parameter $\gamma$ is large and the intensity of noise $(D)$ is small, the quantities $\mathcal{J}(t)$ and $\omega(t)=w-\gamma t$ are slowly varying functions.

Let us introduce the probability density $P(\mathcal{J}, \omega ; t)=\langle\delta(\mathcal{J}$ $-\mathcal{J}(t)) \delta(\omega-\omega(t))\rangle$. Using standard techniques, we find from Eqs. (28) and (29) that the Fokker-Planck equation for $P(\mathcal{J}, \omega ; t)$ has the form

$$
\begin{aligned}
\partial_{t} P= & \frac{12 \eta J^{2}}{\gamma} \frac{\partial}{\partial \mathcal{J}}\left[\mathcal{J} \cos ^{2}(w) P\right] \\
& -\frac{6 \eta J^{2}}{\gamma} \frac{\partial}{\partial \omega}[(\sqrt{\mathcal{J}}+\sin w) \cos (w) P] \\
& +D\left[\frac{\partial}{\partial \mathcal{J}} \sqrt{6 \mathcal{J}} \cos w-\frac{\partial}{\partial \omega} \sqrt{\frac{3}{2}}\left(1+\frac{\sin w}{\sqrt{\mathcal{J}}}\right)\right]^{2} P,
\end{aligned}
$$

where $w=\omega+\gamma t$. The right-hand side of Eq. (30) contains rapidly oscillating functions $[\cos (\omega+\gamma t)$ and $\sin (\omega+\gamma t)]$. We can average this equation with respect to these oscillations (or, in other words, use the Bogolyubov-Mitropolsky averaging procedure; see, e.g. Ref. 26) and obtain

$$
\begin{aligned}
\partial_{t} P= & \frac{6 \eta J^{2}}{\gamma} \frac{\partial}{\partial \mathcal{J}}(\mathcal{J P})+3 D \frac{\partial}{\partial \mathcal{J}}\left(\mathcal{J} \frac{\partial P}{\partial \mathcal{J}}\right) \\
& +\frac{3}{2} D\left(1+\frac{1}{2 \mathcal{J}}\right) \frac{\partial^{2} P}{\partial \omega^{2}} .
\end{aligned}
$$

Equation (31) describes the averaged dynamics of the system under the influence of noise. However, since we are mainly interested in the behavior of the quantity

$$
\left\langle\left|\psi_{0}(t)\right|^{2}\right\rangle=\left\langle|A(t)|^{2}\right\rangle=\left\langle(1+2 q)^{-1 / 2}\right\rangle,
$$

which is measured in our numerical simulations in Sec. II, we do not need to solve Eq. (31). Indeed, it follows from Eq. (26) that

$$
\left\langle(1+2 q)^{-1 / 2}\right\rangle \approx 1-\frac{2 J^{2}}{\gamma^{2}}-\frac{2 J^{2}}{\gamma^{2}}\langle\mathcal{J}\rangle
$$

where

$$
\langle\mathcal{J}\rangle=\int_{0}^{\infty} d \mathcal{J} \int_{0}^{2 \pi} d \omega \mathcal{J P}(\mathcal{J}, \omega) .
$$

Multiplying Eq. (31) by $\mathcal{J}$ and integrating with respect to $\mathcal{J}$ and $\omega$, we obtain

$$
\frac{d}{d t}\langle\mathcal{J}\rangle=-\frac{6 \eta J^{2}}{\gamma}\langle\mathcal{J}\rangle+3 D
$$




$$
\langle\mathcal{J}\rangle=\frac{D \gamma}{2 \eta J^{2}}\left(1-e^{\left(-6 \eta J^{2} / \gamma\right) t}\right),
$$

where the initial condition $\left.\langle\mathcal{J}(t)\rangle\right|_{t=0}=0$ (corresponding to the stationary breather solution in the limit of large $\gamma$ in the absence of noise) was used. Thus, from Eqs. (32) and (33), we finally get

$$
\begin{aligned}
\left\langle\left|\psi_{0}(t)\right|^{2}\right\rangle & \approx 1-\frac{2 J^{2}}{\gamma^{2}}-\frac{D}{\gamma \eta}\left(1-e^{\left(-6 \eta J^{2} / \gamma\right) t}\right) \\
& \approx \begin{cases}1-\frac{2 J^{2}}{\gamma^{2}}-\frac{6 D J^{2}}{\gamma^{2}} t, & \frac{6 \eta J^{2}}{\gamma} t \ll 1, \\
1-\frac{2 J^{2}}{\gamma^{2}}-\frac{D}{\gamma \eta}, & \frac{6 \eta J^{2}}{\gamma} t \gg 1 .\end{cases}
\end{aligned}
$$

We see that the collective-coordinate approach gives a qualitatively good agreement with the results of the numerical simulations from Sec. II as concerns the dependence of the decay rate of the breather on the nonlinearity and noise intensity: The coefficient $6 D J^{2} / \gamma^{2}$ for the linear term in the small-time regime exhibits the same proportionality to $D / \gamma^{2}$ that was observed numerically [compare Figs. 3(a) and 3(b)], and the numerical prefactor is of the same order of magnitude. However, the $\eta$ dependence predicted by Eq. (37) does not agree very well with the numerical results. Indeed, Eq. (37) predicts a stationary nonzero value of the initial-site intensity as $t \rightarrow \infty$ when $\eta \geq D / \gamma$, while we have seen in Sec. II that $\left|\psi_{0}\right|^{2}$ always decays to zero also in this case. The reason for this discrepancy is that in our collectivecoordinate approach we have assumed that the total norm of the breather will be constant in time. However, as is clear from the numerical simulations, in reality radiation will be created through the action of the noise and the norm of the breather (i.e., of the coherent part of the excitation) will decrease with time. Thus relation (11) between the parameters $A$ and $\beta$, which we have assumed to be valid for all times, will in reality only be valid for small $t$.

\section{EXACT RESULTS FOR THE DIMER}

In this section, we will show that if the system described by the DNLS equation (1) is restricted to only contain two sites, it is possible to obtain an exact expression for the stationary distribution of the difference in occupation probability between the sites. We thus rewrite Eq. (1) (assuming rigid boundary conditions) as

$$
\begin{aligned}
& i \dot{\psi}_{1}+J \psi_{2}+\psi_{1}\left(\gamma-\eta \frac{d}{d t}\right)\left|\psi_{1}\right|^{2}+h_{1}(t) \psi_{1}=0, \\
& i \dot{\psi}_{2}+J \psi_{1}+\psi_{2}\left(\gamma-\eta \frac{d}{d t}\right)\left|\psi_{2}\right|^{2}+h_{2}(t) \psi_{2}=0 .
\end{aligned}
$$

To proceed, we change variables as follows:

$$
x(t)=\psi_{1}(t) \psi_{2}^{*}(t)+\psi_{2}(t) \psi_{1}^{*}(t) \equiv \mathcal{N} \sin \theta(t) \cos \phi(t),
$$

$$
y(t)=i\left[\psi_{1}(t) \psi_{2}^{*}(t)-\psi_{2}(t) \psi_{1}^{*}(t)\right] \equiv \mathcal{N} \sin \theta(t) \sin \phi(t),
$$

$$
z(t)=\left|\psi_{1}(t)\right|^{2}-\left|\psi_{2}(t)\right|^{2} \equiv \mathcal{N} \cos \theta(t),
$$

where the parametrization into the two independent variables $\theta$ and $\phi$ is possible due to conservation of the norm (6). From Eqs. (38) and (39), we then find the evolution equations for $\theta$ and $\phi$,

$$
\dot{\phi}=2 J \cos \phi \cot \theta-\gamma \mathcal{N} \cos \theta-2 J \eta \mathcal{N} \sin \theta \sin \phi-f(t),
$$

$$
\dot{\theta}=2 J \sin \theta,
$$

where the noise term $f(t)$ is obtained as

$$
f(t)=h_{1}(t)-h_{2}(t) .
$$

Thus, the original equations (38) and (39) containing multiplicative noise have been reduced to the two equations (43) and (44) containing only additive noise. From Eqs. (2) and (45), we find the autocorrelation function

$$
\left\langle f(t) f\left(t^{\prime}\right)\right\rangle=4 D \delta\left(t-t^{\prime}\right) .
$$

Using standard techniques, we can then derive the Fokker-Planck equation for the probability density $P(\theta, \phi ; t)$ $=\langle\delta(\theta-\theta(t)) \delta(\phi-\phi(t))\rangle$. It reads

$$
\begin{aligned}
\partial_{t} P= & -2 J \sin \phi \partial_{\theta} P-\partial_{\phi}[(2 J \cos \phi \cot \theta-\gamma \mathcal{N} \cos \theta \\
& -2 J \eta \mathcal{N} \sin \theta \sin \phi) P]+2 D \partial_{\phi}^{2} P .
\end{aligned}
$$

By replacing the left-hand side with 0 , we find its stationary solution $P_{s}(\theta, \phi)$ (assuming $J \neq 0$ ),

$$
\begin{aligned}
P_{s}(\theta, \phi)= & C \sin \theta \exp \left\{\frac{J}{k_{B} T}(\mathcal{N} \sin \theta \cos \phi\right. \\
& \left.\left.+\frac{\gamma \mathcal{N}^{2}}{4 J} \cos ^{2} \theta\right)\right\},
\end{aligned}
$$

where $C$ is a normalization constant and the relation $D / \eta=k_{B} T$ from Eq. (5) has been used. Here we should remark that this solution was also found in Ref. 18 as a solution to the approximate Fokker-Planck equation derived for the coupled dimer equations (3) and (4) in the limit of large damping $\lambda$. By integrating over $\phi$ and returning to the variable $z=\mathcal{N} \cos \theta$, we finally obtain the stationary distribution function for the difference in the occupation probability between the sites as

$$
\mathcal{P}(z)=\bar{C} \exp \left(\frac{\gamma z^{2}}{4 k_{B} T}\right) I_{0}\left(\frac{J \sqrt{\mathcal{N}^{2}-z^{2}}}{k_{B} T}\right), \quad-\mathcal{N} \leqslant z \leqslant \mathcal{N},
$$

where $\bar{C}$ is a new normalization constant and $I_{0}$ is a modified Bessel function of the first kind. ${ }^{27}$

From Eq. (49), we observe that the shape of the stationary probability distribution $\mathcal{P}(z)$ changes qualitatively when varying the rescaled parameters $\widetilde{\gamma}$ and $\widetilde{T}$ defined as

$$
\tilde{\gamma}=\frac{\gamma \mathcal{N}}{2 J}, \quad \widetilde{T}=\frac{k_{B} T}{J \mathcal{N}} .
$$

Three different regions in the $\widetilde{\gamma}-\widetilde{T}$ plane can be identified (see Fig. 4). In region I, where $\widetilde{\gamma}<I_{1}(1 / \widetilde{T}) / I_{0}(1 / \widetilde{T})$, the probability distribution $\mathcal{P}(z)$ is unimodal, having one single 


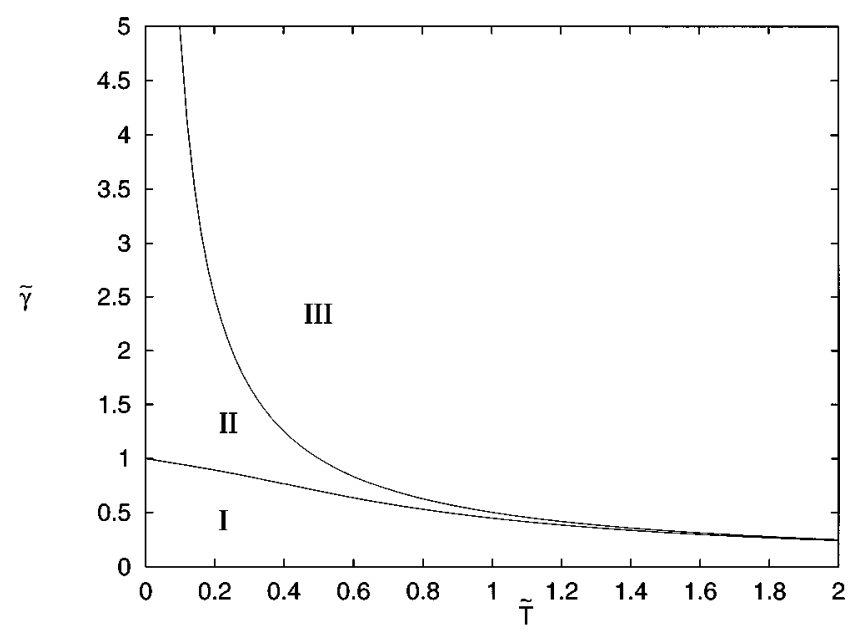

FIG. 4. Phase diagram for the probability distribution $\mathcal{P}(z)$ from Eq. (49). In region $\mathrm{I}, \mathcal{P}(z)$ has a single maximum at $z=0$; in region II, $\mathcal{P}(z)$ has two maxima for $0<|z|<\mathcal{N}$; and in region III, $\mathcal{P}(z)$ attains its maximum value at $z= \pm \mathcal{N}$.

maximum at $z=0$ corresponding to equal population between the sites. When $I_{1}(1 / \widetilde{T}) / I_{0}(1 / \widetilde{T})<\widetilde{\gamma}<1 / 2 T$ (region II), the probability distribution becomes bimodal, having two equivalent maxima corresponding to an excess of population on either of the sites and a local minimum at $z=0$. The two maxima are obtained as solutions to the equation $I_{1}(b) / b I_{0}(b)=\widetilde{\gamma} \widetilde{T}$, where $b=(1 / \widetilde{T}) \sqrt{1-(z / \mathcal{N})^{2}}$. As $\widetilde{\gamma}$ (or $\widetilde{T})$ increases in this region, the two maxima move outwards towards $z= \pm \mathcal{N}$, and for $\widetilde{\gamma}>1 / 2 \widetilde{T}$ (region III), the probability distribution attains its maximum value at the end points $z$ $= \pm \mathcal{N}$.

It is instructive to compare the nature of the noise-induced phase transition described above with the self-trapping transition that occurs in the noise-free dimer system. In the latter case there is a static transition at $\tilde{\gamma}=1$, since, if and only if $\tilde{\gamma}>1$, there exist stationary states where the two sites are unequally populated. ${ }^{5}$ The difference in occupation probability is given by $z= \pm \mathcal{N} \sqrt{1-1 / \widetilde{\gamma}^{2}}$. There is also a dynamic self-trapping transition occurring at $\tilde{\gamma}=2$ in the undamped system $(\eta=0)$; i.e., if one single site is initially excited, the main part of the intensity will remain at this site if and only if $\tilde{\gamma}>2{ }^{28}$ When $\tilde{\gamma}<2$, the variable $z$ will perform oscillations described by Jacobi elliptic functions between $-\mathcal{N}$ and $\mathcal{N}$. In the presence of damping, the system will always settle down in one of the self-trapped stationary states when $\tilde{\gamma}>1$, except when the two sites are initially identically excited. However, the final self-trapped state will not necessarily have its main intensity at the initially excited site when $1<\widetilde{\gamma}<2$, since the variable $z$ may exhibit an initial oscillatory behavior if the damping is small.

Thus we see that since the location of the transition from a unimodal (region I) to bimodal (region II) probability distribution $\mathcal{P}(z)$ approaches the value $\tilde{\gamma}=1$ as $\widetilde{T} \rightarrow 0$, it coincides with the transition into a self-trapped state in the noisefree dimer. However, it is important to remember that when $T>0$, the state will not necessarily be trapped around one of the probability maxima even if the distribution is bimodal, since the noise may induce frequent transitions between the sites. An estimate of the tendency for the quasiparticle to "jump" between the sites can be obtained by considering the quantity $\mathcal{P}(0) / \mathcal{P}\left(z_{\max }\right)$, where $z_{\max }$ is the location of the probability maximum. For large $\widetilde{\gamma}$, this quantity is seen from Eq. (49) to behave approximately as $e^{-\tilde{\gamma} / 2 \widetilde{T}}$. Thus, when $\widetilde{T}$ is small compared to $\widetilde{\gamma}$ (i.e., when $D / \eta \ll \gamma \mathcal{N}^{2} / 4$ ), the probability to find the quasiparticle equally distributed between the sites will be very small and, consequently, also the probability to make "jumps" between the sites. An excitation initially localized on one of the sites will stay there practically forever, the self-trapping will be preserved, and the twopeaked equilibrium probability distribution will in practice never be reached. On the other hand, when $\widetilde{T} \sim \widetilde{\gamma} / 2$, the probability distribution is relatively flat, which means that the noise will be strong enough to cause transitions between the sites, and the self-trapping is destroyed. This behavior has been confirmed with numerical simulations, and it also agrees with similar conclusions obtained for the model studied in Ref. 18.

\section{CONCLUSIONS}

We have found that introducing multiplicative white noise and nonlinear damping into the discrete nonlinear Schrödinger equation will cause decay of the self-trapped discrete breathers which are created for large nonlinearities. A numerical analysis showed that the intensity at the central breather site would initially decrease approximately linearly with time. The decay rate was found to decrease with increasing nonlinearity $\gamma$ and with increasing damping $\eta$, and increase with increasing noise variance $D$. Using a collective coordinate approach with an exponentially localized trial function, the linear decay was predicted and a decay rate proportional to $D / \gamma^{2}$ for small $D$ and large $\gamma$ was obtained in good agreement with the numerical results. However, not taking into account the gradual decrease of the norm for the breather, the collective-coordinate method could not predict its final destruction. Indeed, for $\eta$ large enough the collective-coordinate method predicted a stationary nonzero mean value of the initial-site intensity in the long-time limit, while the numerical calculations showed that for all parameter values the breather would finally be destroyed and the long-time behavior would be diffusive.

In the particular case of the dimer, we showed that, by performing a suitable change of variables, the original equations containing multiplicative noise could be transformed into equations containing only additive noise. The FokkerPlanck equation for this system could then be derived, and its stationary solution was found exactly. By analyzing this solution, we found that by increasing the temperature $T \sim D / \eta$, a transition from a unimodal phase, corresponding to maximum probability for an equal population between the sites, to a bimodal phase, corresponding to maximum probability for an excess of population on either of the two sites, could be achieved. We also found that the self-trapping for the dimer case would in practice be preserved as long as $T \ll \gamma$, since the probability for transitions between the sites in this case would be exponentially small.

It is interesting to compare the results obtained for the infinite DNLS chain with the results obtained for the dimer. In the case of the infinite chain, we found that the selftrapped breather cannot exist as a stationary state for any 
nonzero noise variance, while in the dimer case the selftrapping will survive for weak noise (even though the noise might cause the quasiparticle to occasionally jump between the sites, equipartition between the sites will never be obtained). The origin of these different types of behavior is that for the dimer no external degrees of freedom can be excited, so that the total norm of the excitation on the two sites must be conserved. On the other hand, in the infinite chain the noise will gradually destroy the coherence of the breather and create linear radiation into the rest of the lattice, thereby reducing the norm of the breather (or, equivalently, reducing the effective nonlinearity).

\section{ACKNOWLEDGMENTS}

We would like to thank V. K. Mezentsev and J. Juul Rasmussen for discussions. Yu.B.G. acknowledges support from the Technical University of Denmark and the support of Ukrainian Government and International Science Foundation under Grant No. 59100. M.J. acknowledges financial support from the Swedish Foundation for International Cooperation in Research and Higher Education. The present work was supported by the Danish Research Council through Contract No. 9313393 (supercomputing projects).
${ }^{1}$ A. J. Sievers and S. Takeno, Phys. Rev. Lett. 61, 970 (1988).

${ }^{2}$ S. Aubry, Physica D 71, 196 (1994); R. S. MacKay and S. Aubry, Nonlinearity 7, 1623 (1994).

${ }^{3}$ S. Flach, Phys. Rev. E 50, 3134 (1994); 51, 1503 (1995); 51, 3579 (1995).

${ }^{4}$ D. Hennig, N. G. Sun, H. Gabriel, and G. P. Tsironis, Phys. Rev. E 52, 255 (1995).

${ }^{5}$ A. C. Scott and L. MacNeil, Phys. Lett A 98, 87 (1983); J. C. Eilbeck, P. S. Lomdahl, and A. C. Scott, Physica D 16, 318 (1985).

${ }^{6}$ M. I. Molina and G. P. Tsironis, Physica D 65, 267 (1993).

${ }^{7}$ L. J. Bernstein, K. W. DeLong, and N. Finlayson, Phys. Lett. A 181, 135 (1993).

${ }^{8}$ T. Holstein, Ann. Phys. (N.Y.) 8, 325 (1959); D. Emin and T. Holstein, Phys. Rev. Lett. 36, 323 (1976).

${ }^{9}$ A. S. Davydov, Solitons in Molecular Systems (Reidel, Dordrecht, 1985); in Davydov's Soliton Revisited, edited by P. L. Christiansen and A. C. Scott (Plenum, New York, 1990).

${ }^{10}$ S. M. Jensen, IEEE J. Quantum Electron. QE-18, 1580 (1982); D. N. Christodoulides and R. I. Joseph, Opt. Lett. 13, 794 (1988); A. B. Aceves, C. De Angelis, T. Peschel, R. Muschall, F. Lederer, S. Trillo, and S. Wabnitz, Phys. Rev. E 53, 1172 (1996).

${ }^{11}$ O. Bang, J. J. Rasmussen, and P. L. Christiansen, Physica D 68 , 169 (1993); Nonlinearity 7, 205 (1994).

${ }^{12}$ E. W. Laedke, K. H. Spatschek, and S. K. Turitsyn, Phys. Rev. Lett. 73, 1055 (1994).

${ }^{13}$ P. L. Christiansen, Yu. B. Gaididei, K. Ø. Rasmussen, V. K. Mezentsev, and J. Juul Rasmussen, Phys. Rev. B 54, 900 (1996).

${ }^{14}$ J. Juul Rasmussen and K. Rypdal, Phys. Scr. 33, 481 (1986).

${ }^{15}$ G. C. Huth, F. Gutmann, and G. Vitiello, Phys. Lett. A 140, 339 (1989); P. L. Christiansen, S. Pagano, and G. Vitiello, ibid. 154, 381 (1991); P. L. Christiansen, O. Bang, S. Pagano, and G. Vitiello, Nanobiology 1, 229 (1992).

${ }^{16}$ O. Bang, P. L. Christiansen, F. If, K. Ф. Rasmussen, and Yu. B. Gaididei, Phys. Rev. E 49, 4627 (1994).

${ }^{17}$ P. S. Lomdahl and W. C. Kerr, Phys. Rev. Lett. 55, 1235 (1985); A. F. Lawrence, J. C. McDaniel, D. B. Chang, B. M. Pierce, and
R. R. Birge, Phys. Rev. A 33, 1188 (1986); W. Förner, J. Phys. Condens. Matter 3, 4333 (1991).

${ }^{18}$ P. Grigolini, H.-L. Wu, and V. M. Kenkre, Phys. Rev. B 40, 7045 (1989); V. M. Kenkre and P. Grigolini, Z. Phys. B 90, 247 (1993); V. M. Kenkre, Physica D 68, 153 (1993); V. M. Kenkre and M. Kus, Phys. Rev. B 49, 5956 (1994).

${ }^{19}$ P. L. Christiansen, Yu. B. Gaididei, M. Johansson, K. Ф. Rasmussen, and I. I. Yakimenko, Phys. Rev. E 54, 924 (1996).

${ }^{20}$ M. Johansson, M. Hörnquist, and R. Riklund, Phys. Rev. B 52, 231 (1995).

${ }^{21}$ L. Lapidus and J. H. Seinfeld, Numerical Solution of Ordinary Differential Equations (Academic, New York, 1971), and references therein.

${ }^{22}$ The method used is designed to give the Stratonovich solution to a stochastic differential equation. We note that since the noise term in Eq. (1) is multiplicative, this solution will in general be different from solutions defined in other ways, e.g., the Ito solution (see, e.g., Ref. 23). However, since integration rules from standard calculus were used to derive Eq. (1), the Stratonovich solution is the appropriate one here.

${ }^{23}$ T. C. Gard, Introduction to Stochastic Differential Equations (Dekker, New York, 1988), and references therein.

${ }^{24}$ D. Anderson, M. Bonnedal, and M. Lisak, Phys. Fluids 22, 1838 (1979); M. Desaix, D. Anderson, and M. Lisak, Phys. Rev. A 40, 2441 (1989); J. Opt. Soc. Am. B 8, 2082 (1991).

${ }^{25}$ O. Bang, P. L. Christiansen, F. If, K. Ø. Rasmussen, and Yu. B. Gaididei, Appl. Anal. 57, 3 (1995); K. Ø. Rasmussen, Yu. B. Gaididei, O. Bang, and P. L. Christiansen, Phys. Lett. A 204, 121 (1995).

${ }^{26}$ J. Kevorkian and J. D. Cole, Perturbation Methods in Applied Mathematics (Springer-Verlag, New York, 1985).

${ }^{27}$ Handbook of Mathematical Functions, edited by M. Abramowitz and I. A. Stegun (Dover, New York, 1965).

${ }^{28}$ V. M. Kenkre and D. K. Campbell, Phys. Rev. B 34, 4959 (1986); V. M. Kenkre and G. P. Tsironis, ibid. 35, 1473 (1987); G. P. Tsironis and V. M. Kenkre, Phys. Lett. A 127, 209 (1988); V. M. Kenkre and H.-L. Wu, Phys. Rev. B 39, 6907 (1989). 